ANNALES

POLONICI MATHEMATICI

LXXVII.2 (2001)

\title{
Sequences of differential operators: exponentials, hypercyclicity and equicontinuity
}

\author{
by L. Bernal-González and J. A. Prado-Tendero (Sevilla)
}

\begin{abstract}
An eigenvalue criterion for hypercyclicity due to the first author is improved. As a consequence, some new sufficient conditions for a sequence of infinite order linear differential operators to be hypercyclic on the space of holomorphic functions on certain domains of $\mathbb{C}^{N}$ are shown. Moreover, several necessary conditions are furnished. The equicontinuity of a family of operators as above is also studied, and it is characterized if the domain is $\mathbb{C}^{N}$. The results obtained extend or improve earlier work of several authors.
\end{abstract}

1. Introduction, notation and preliminary results. Throughout this paper we denote by $\mathbb{N}$ the set of positive integers, by $\mathbb{R}$ the real line, by $\mathbb{C}$ the field of complex numbers, and by $\mathbb{N}_{0}$ the set $\mathbb{N}_{0}=\mathbb{N} \cup\{0\}$. Let $X, Y$ be two linear topological spaces, $T_{i}: X \rightarrow Y(i \in I:=$ an arbitrary index set) a family of continuous linear mappings, and $x \in X$. Then $x$ is said to be hypercyclic or universal for $\left(T_{i}\right)$ whenever its orbit $\left\{T_{i} x: i \in I\right\}$ under $\left(T_{i}\right)$ is dense in $Y$. The family $\left(T_{i}\right)$ is called hypercyclic whenever it has a hypercyclic vector. Note that if $\left(T_{i}\right)$ is hypercyclic then it is not equicontinuous, but the converse is false in general. In the case $I=\mathbb{N}$, it is clear that, in order that a sequence $\left(T_{n}\right)$ can be hypercyclic, $Y$ must be separable. If $T: X \rightarrow X$ is an operator (= continuous linear selfmapping) on $X$, then a vector $x \in X$ is said to be hypercyclic for $T$ if it is hypercyclic for the sequence $\left(T^{n}\right)$ of iterates of $T$, i.e., $T^{n}=T \circ \ldots \circ T$ ( $n$-fold). The operator $T$ is hypercyclic when there is a hypercyclic vector for $T$. The symbols $\mathrm{HC}(T)$ and $\operatorname{HC}\left(\left(T_{i}\right)\right)$ will denote, respectively, the set of hypercyclic vectors of an operator $T$ and of a family $T_{i}: X \rightarrow Y(i \in I)$ of continuous linear mappings. In the last two

2000 Mathematics Subject Classification: Primary 47B38; Secondary 30E10, 47A16, 47E05, 47F05.

Key words and phrases: hypercyclic operators and sequences, equicontinuous family, infinite order linear differential operator, subexponential and exponential type, eigenvalue criterion, total subset, exponential functions, Runge domain, polydomain.

This work has been supported in part by D.G.E.S. PB96-1348 and the Junta de Andalucía. 
decades an extensive literature about hypercyclicity has been developed; a good survey is [Gr1].

Let $G$ be a nonempty open subset of $\mathbb{C}^{N}(N \in \mathbb{N})$. We say that $G$ is a domain when, in addition, it is connected. A domain $G \subset \mathbb{C}^{N}$ is said to be a Runge domain (see [Hor] or [Kra]) if each holomorphic function on $G$ can be uniformly approximated by polynomials on compact subsets of $G$. Note that, if $N=1$, then $G$ is a Runge domain if and only if it is simply connected. By $H(G)$ we denote, as usual, the Fréchet space of holomorphic functions on $G$, endowed with the compact-open topology. Recall that the family $\{V(K, \varepsilon): \varepsilon>0, K$ is a compact subset of $G\}$ is a neighbourhood basis for the origin in $H(G)$. Here $V(K, \varepsilon):=\left\{f \in H(G):\|f\|_{K}<\varepsilon\right\}$. For $A \subset \mathbb{C}^{N}$ we have denoted $\|g\|_{A}:=\sup \{|g(z)|: z \in A\}$ whenever $g$ is a complex function defined on the set $A$.

G. Godefroy and J. H. Shapiro [GoS, Section 5] proved in 1991 the following generalization of the classical theorems on approximation by translates and derivatives of a single entire function due respectively to Birkhoff [Bir] and MacLane [Mac]: If $T$ is an operator on the space $H\left(\mathbb{C}^{N}\right)$ of entire functions on $\mathbb{C}^{N}$ that commutes with each of the translation operators $\tau_{a}$ $\left(a \in \mathbb{C}^{N}\right)$ given by $\tau_{a} f(z)=f(z+a)\left(f \in H\left(\mathbb{C}^{N}\right), z \in \mathbb{C}^{N}\right)$, and is not a scalar multiple of the identity, then $\operatorname{HC}(T)$ is a dense $G_{\delta^{-}}$subset of $H\left(\mathbb{C}^{N}\right)$; in addition, $\mathrm{HC}(T)$ contains all nonzero functions of a dense, $T$-invariant, linear submanifold of $X:=H\left(\mathbb{C}^{N}\right)$. P. Bourdon [Bou] and D. Herrero [Her] proved independently that every hypercyclic operator $T$ on any Banach space $X$ (in fact, on any real or complex locally convex space $X$; see [Ans] and $[\mathrm{Bes}])$ has the same property. The first author of the present paper has recently shown [Be4] that if $X$ and $Y$ are two separable metrizable linear topological spaces and if $T_{n}: X \rightarrow Y(n \in \mathbb{N})$ is a sequence of continuous linear mappings for which there is an increasing sequence $\left(n_{j}\right)$ of positive integers with the property that $\mathrm{HC}\left(\left(T_{m_{j}}\right)\right)$ is dense for every subsequence $\left(m_{j}\right)$ of $\left(n_{j}\right)$, then $\mathrm{HC}\left(\left(T_{n}\right)\right) \cup\{0\}$ contains a dense linear submanifold of $X$.

Given $N \in \mathbb{N}$, denote by $D_{j}(1 \leq j \leq N)$ complex partial differentiation with respect to the $j$ th coordinate. A multi-index is an $N$-tuple $p=\left(p_{1}, \ldots, p_{N}\right)$ of nonnegative integers. Set $|p|=p_{1}+\ldots+p_{N}, p !=$ $p_{1} ! \ldots p_{N} !, D^{p}=D_{1}^{p_{1}} \circ \ldots \circ D_{N}^{p_{N}}$ (with $D_{j}^{0}=I=$ the identity operator for every $j \in\{1, \ldots, N\})$, and $|z|=\left(\left|z_{1}\right|^{2}+\ldots+\left|z_{N}\right|^{2}\right)^{1 / 2}, z^{p}=z_{1}^{p_{1}} \ldots z_{N}^{p_{N}}$, $z w=z_{1} w_{1}+\ldots+z_{N} w_{N}$ if $z=\left(z_{1}, \ldots, z_{N}\right), w=\left(w_{1}, \ldots, w_{N}\right)$. An entire function $\Phi(z)=\sum_{|p| \geq 0} a_{p} z^{p}$ is said to be of exponential type whenever there exist positive constants $A$ and $B$ such that $|\Phi(z)| \leq A e^{B|z|}$ $\left(z \in \mathbb{C}^{N}\right)$. For later reference, we denote by $\mathcal{E}$ the class of all entire functions of exponential type. An entire function $\Phi$ is said to be of subexponential type if, given $\varepsilon>0$, there is a positive constant $A=A(\varepsilon)$ such that 
$|\Phi(z)| \leq A e^{\varepsilon|z|}\left(z \in \mathbb{C}^{N}\right)$. Every entire function of subexponential type is obviously in $\mathcal{E}$. It is easy to realize (see, for instance, [Val], [Dic] or [Be3]) that if $G \subset \mathbb{C}^{N}$ is a nonempty open subset and $\Phi$ is an entire function as above of subexponential type, then the series $\Phi(D)=\sum_{|p| \geq 0} a_{p} D^{p}$ defines an operator on $H(G)$. If $G=\mathbb{C}^{N}$, the same result holds just upon assuming that $\Phi$ is of exponential type. So $\Phi(D)$ defines, under the latter conditions, an infinite order linear differential operator with constant coefficients. It is shown in $[\mathrm{GoS}]$ that an operator $L$ on $H\left(\mathbb{C}^{N}\right)$ commutes with every translation operator $\tau_{a}\left(a \in \mathbb{C}^{N}\right)$ if and only if $L$ commutes with each $D_{k}(1 \leq k \leq N)$ if and only if $L=\Phi(D)$ for some entire function $\Phi$ in $\mathcal{E}$.

As a consequence of an eigenvalue criterion for hypercyclicity [Be3, Theorem 7], the first author obtained some extensions of Godefroy-Shapiro's result [Be3, Theorems 8-9], this time about the hypercyclicity of a sequence of operators $\left(\Phi_{n}(D)\right)$ defined on the space of holomorphic functions on a Runge domain $G$ of $\mathbb{C}^{N}$. Furthermore, conditions about the equicontinuity of a sequence $\left(c_{n} D^{n}\right)$, where $\left(c_{n}\right) \subset \mathbb{C}$ (note that this is the special case $\Phi_{n}(z)=c_{n} z^{n}$ ), are shown in [Be1] and [Be2] (see also [Cal], where each $c_{n}$ is replaced by a holomorphic fuction $\left.c_{n}(z)\right)$.

Our aim in this paper is to provide with a more general eigenvalue criterion and, as a consequence, new sufficient conditions for the hypercyclicity of a sequence of infinite order linear differential operators. In addition, necessary conditions are established, and some special cases are analyzed. Necessary conditions and sufficient conditions for the equicontinuity of the sequence are also furnished, and in particular we completely characterize the equicontinuity in $H\left(\mathbb{C}^{N}\right)$.

\section{Eigenvalues, exponentials, hypercyclicity and equicontinuity.} As in [GoS, Section 5] and [Be3, Theorems 8-9], the key to the proof of hypercyclicity is to provide a good supply of eigenvectors of the corresponding operators. Recall that, in a linear topological space, a subset is said to be total whenever its linear span is dense. If $T$ is an operator and $e$ is an eigenvector, then we denote by $\lambda(T, e)$ its corresponding eigenvalue. Next, we state as a lemma the following rather general hypercyclicity criterion, which can be found in [Gr1].

Lemma 2.1. Assume that $X$ is a Baire topological vector space, $Y$ is a separable metrizable topological vector space and $T_{n}: X \rightarrow Y(n \in \mathbb{N})$ are continuous linear mappings. Suppose that there are dense subsets $X_{0}$ of $X$ and $Y_{0}$ of $Y$ and mappings $S_{n}: Y_{0} \rightarrow X$ such that

(a) for every $x \in X_{0}$, there exists an increasing sequence $\left(n_{k}\right)$ of positive integers with $T_{n_{k}} x \rightarrow 0(k \rightarrow \infty)$, 
(b) for every $y \in Y_{0},\left(S_{n} y\right)$ converges, and

(c) for every $y \in Y_{0}, T_{n}\left(S_{n} y\right) \rightarrow y(n \rightarrow \infty)$.

Then $\mathrm{HC}\left(\left(T_{n}\right)\right)$ is residual.

As noted in [Gr1, Remark 2], if all the limits in (b) are zero then we may weaken (a) to: for every $x \in X_{0}$, there exists an increasing sequence $\left(n_{k}\right)$ of positive integers such that $\left(T_{n_{k}} x\right)$ converges. Furthermore, the quantifier " $\exists\left(n_{k}\right)$ " can be shifted from (a) to (b) or (c).

Under the same hypothesis for $X$ and $Y$, it can be proved (see, for instance, $[\mathrm{Be} 2])$ that the following condition is also sufficient in order that $\mathrm{HC}\left(\left(T_{n}\right)\right)$ be residual: there exist dense subsets $X_{0}$ of $X$ and $Y_{0}$ of $Y$ with the property that for every $x \in X_{0}$ and every $y \in Y_{0}$ there exists an increasing sequence $\left(n_{k}\right)$ of positive integers and a sequence $\left(x_{k}\right) \subset X$ such that $x_{k} \rightarrow 0$, $T_{n_{k}} x \rightarrow 0$ and $T_{n_{k}} x_{n_{k}} \rightarrow y$ as $k \rightarrow \infty$.

By using the latter result, the next eigenvalue criterion can be proved (see [Be3, Theorem 7]): Let $X$ be a separable $F$-space and $\left(T_{n}\right)$ a sequence of operators on $X$. Assume that there are two total subsets $\mathcal{A}, \mathcal{B}$ of $X$ with the property that for every pair of finite subsets $\mathcal{F}_{1} \subset \mathcal{A}$ and $\mathcal{F}_{2} \subset \mathcal{B}$ there is an increasing sequence $\left(n_{k}\right)$ in $\mathbb{N}$ such that every element in $\mathcal{F}_{1} \cup \mathcal{F}_{2}$ is an eigenvector for each $T_{n_{k}}$, and $\lambda\left(T_{n_{k}}, a\right) \rightarrow 0(k \rightarrow \infty)$ for all $a \in \mathcal{F}_{1}$ and $\lambda\left(T_{n_{k}}, b\right) \rightarrow \infty(k \rightarrow \infty)$ for all $b \in \mathcal{F}_{2}$. Then $\mathrm{HC}\left(\left(T_{n}\right)\right)$ is residual.

If we employ Lemma 2.1 (and the note after it) instead of the result just mentioned then the following eigenvalue criterion can be obtained. The proof is left to the interested reader.

TheOREM 2.2. Let $X$ be a separable $F$-space and $\left(T_{n}\right)$ be a sequence of operators on $X$. Assume that there are two total subsets $\mathcal{A}, \mathcal{B}$ of $X$ satisfying at least one of the following conditions:

(A) For every finite subset $\mathcal{F} \subset \mathcal{A}$ there is an increasing sequence $\left(n_{k}\right)$ in $\mathbb{N}$ such that every element in $\mathcal{F}$ is an eigenvector for each $T_{n_{k}}$ and $\lambda\left(T_{n_{k}}, a\right) \rightarrow 0(k \rightarrow \infty)$ for all $a \in \mathcal{F}$. In addition, every element in $\mathcal{B}$ is an eigenvector for each $T_{n}$ and for every $b \in \mathcal{B}$ the sequence $\left(\lambda\left(T_{n}, b\right)\right)$ converges to a nonzero scalar.

(B) For every finite subset $\mathcal{F} \subset \mathcal{A}$ there is an increasing sequence $\left(n_{k}\right)$ in $\mathbb{N}$ such that for every $a \in \mathcal{F}$ the sequence $\left(\lambda\left(T_{n_{k}}, a\right)\right)$ converges. In addition, every element in $\mathcal{B}$ is an eigenvector for each $T_{n}$ and $\lambda\left(T_{n}, b\right) \rightarrow \infty$ $(n \rightarrow \infty)$ for every $b \in \mathcal{B}$.

(C) Every element in $\mathcal{A}$ is an eigenvector for each $T_{n}$ and $\lambda\left(T_{n}, a\right) \rightarrow 0$ $(n \rightarrow \infty)$ for every $a \in \mathcal{A}$. In addition, for every finite subset $\mathcal{F} \subset \mathcal{B}$ there is an increasing sequence $\left(n_{k}\right)$ in $\mathbb{N}$ such that every element in $\mathcal{F}$ is an eigenvector for each $T_{n_{k}}$ and for every $b \in \mathcal{F}$ the sequence $\left(\lambda\left(T_{n_{k}}, b\right)\right)$ converges to a nonzero scalar. 
(D) Every element in $\mathcal{A}$ is an eigenvector for each $T_{n}$ and for every $a \in \mathcal{A}$ the sequence $\left(\lambda\left(T_{n}, a\right)\right)$ converges. In addition, for every finite subset $\mathcal{F} \subset \mathcal{B}$ there is an increasing sequence $\left(n_{k}\right)$ in $\mathbb{N}$ such that every element in $\mathcal{F}$ is an eigenvector for each $T_{n_{k}}$ and $\left(\lambda\left(T_{n_{k}}, b\right)\right) \rightarrow \infty(k \rightarrow \infty)$ for every $b \in \mathcal{F}$.

Then $\mathrm{HC}\left(\left(T_{n}\right)\right)$ is residual.

In another order of ideas, recall that $\mathcal{E}$ denotes the class of entire functions on $\mathbb{C}^{N}$ of exponential type. We say that a subset $S \subset \mathbb{C}^{N}$ is an $\mathcal{E}$-unicity set whenever the following property holds: if $f \in \mathcal{E}$ and $f(z)=0$ for all $z \in S$ then $f \equiv 0$. Note that, by the identity principle for holomorphic functions, if $f$ is an arbitrary entire function vanishing on $S$ and $S$ is a nonempty open set (or even just a set with at least one accumulation point if $N=1$ ) then $f \equiv 0$. This property of $S$ is not necessary for the class $\mathcal{E}$; for instance, if $N=1$ and $\chi:=\limsup _{r \rightarrow \infty} \log n(r) / \log r>1$, where $n(r)$ is the number of points of $S \cap\{|z| \leq r\}$, then $S$ is an $\mathcal{E}$-unicity set (e.g., $S=\left\{n^{1 / 2}: n \in \mathbb{N}\right\}$, which gives $\chi=2$ ). Indeed, if $f \not \equiv 0$, the latter condition would imply that the convergence exponent of the sequence of zeros of $f$ is strictly greater that the growth order of $f$, which is clearly impossible. The next lemma will be useful later. Its proof is classical, but we include it for the sake of completeness. If $c \in \mathbb{C}^{N}$ then we write $e_{c}(z)=\exp (c z)$.

Lemma 2.3. If $S$ is an $\mathcal{E}$-unicity set then $M(S):=\left\{e_{c}: c \in S\right\}$ is total in $H\left(\mathbb{C}^{N}\right)$.

Proof. Fix a functional $L \in H\left(\mathbb{C}^{N}\right)^{*}$ (= the topological dual space of $\left.H\left(\mathbb{C}^{N}\right)\right)$ such that $L\left(e_{c}\right)=0$ for all $c \in S$. Consider the Laplace transform $\widetilde{L}$ of $L$ (see [Hor, p. 100]) given by $\widetilde{L}(z)=L\left(e_{z}\right)\left(z \in \mathbb{C}^{N}\right)$. Then it is easy to show that $\widetilde{L}$ is an entire function on $\mathbb{C}^{N}$ of exponential type which vanishes at $S$. Since $S$ is an $\mathcal{E}$-unicity set, we get $\widetilde{L} \equiv 0$. Then $\left(D^{p} \widetilde{L}\right)(0)=0$ for all $p \in \mathbb{N}_{0}^{N}$. But it is easy to show by induction that $\left(D^{p} \widetilde{L}\right)(0)=L\left(\alpha_{p}\right)$, where $\alpha_{p}(t)=t^{p}\left(t \in \mathbb{C}^{N}\right)$. By linearity, $L$ vanishes at every polynomial, so $L \equiv 0$ because the set of polynomials is dense in $H\left(\mathbb{C}^{N}\right)$. Summarizing, if $L(f)=0$ for all $f \in M(S)$ then $L(f)=0$ for all $f \in H\left(\mathbb{C}^{N}\right)$. By the Hahn-Banach theorem, the linear span of $M(S)$ is dense in $H\left(\mathbb{C}^{N}\right)$ or, equivalently, $M(S)$ is total.

Next, we state eight conditions that may or may not be satisfied by a sequence $\left(\Phi_{n}\right) \subset H\left(\mathbb{C}^{N}\right)$. Recall that if $\Phi(z)=\sum_{|p| \geq 0} a_{p} z^{p} \in H\left(\mathbb{C}^{N}\right)$ and $\Phi$ is not identically zero, its multiplicity for the zero at the origin is $m(\Phi)=\min \left\{|p|: a_{p} \neq 0\right\}$. Note that $\Phi(D) e_{c}=\Phi(c) e_{c}$ for all $c \in \mathbb{C}^{N}$, so $e_{c}$ is an eigenvector of $\Phi(D)$ with eigenvalue $\Phi(c)$.

(P) There are two $\mathcal{E}$-unicity sets $A, B$ in $\mathbb{C}^{N}$ such that for every pair of finite subsets $F_{1} \subset A$ and $F_{2} \subset B$ there exists an increasing sequence 
$\left(n_{k}\right) \subset \mathbb{N}$ with $\Phi_{n_{k}}(a) \rightarrow 0(k \rightarrow \infty)$ for all $a \in F_{1}$ and $\Phi_{n_{k}}(b) \rightarrow \infty$ $(k \rightarrow \infty)$ for all $b \in F_{2}$.

(Q) There is an $\mathcal{E}$-unicity set $B$ in $\mathbb{C}^{N}$ such that for every finite subset $F \subset B$ there exists an increasing sequence $\left(n_{k}\right) \subset \mathbb{N}$ with $m\left(\Phi_{n_{k}}\right) \rightarrow \infty$ $(k \rightarrow \infty)$ and $\Phi_{n_{k}}(b) \rightarrow \infty(k \rightarrow \infty)$ for all $b \in F$.

(R) There are two $\mathcal{E}$-unicity sets $A, B$ in $\mathbb{C}^{N}$ such that for every finite subset $F \subset A$ there exists an increasing sequence $\left(n_{k}\right) \subset \mathbb{N}$ with $\Phi_{n_{k}}(a) \rightarrow 0$ $(k \rightarrow \infty)$ for all $a \in F$, and for each $b \in B$ the sequence $\left(\Phi_{n}(b)\right)$ converges to a nonzero complex number.

(S) There is an $\mathcal{E}$-unicity set $B$ in $\mathbb{C}^{N}$ such that for each $b \in B$ the sequence $\left(\Phi_{n}(b)\right)$ converges to a nonzero complex number, and there exists an increasing sequence $\left(n_{k}\right) \subset \mathbb{N}$ with $m\left(\Phi_{n_{k}}\right) \rightarrow \infty(k \rightarrow \infty)$.

(T) There are two $\mathcal{E}$-unicity sets $A, B$ in $\mathbb{C}^{N}$ such that for every finite subset $F \subset A$ there exists an increasing sequence $\left(n_{k}\right) \subset \mathbb{N}$ with the property that for every $a \in F$ the sequence $\left(\Phi_{n_{k}}(a)\right)$ converges. In addition, $\Phi_{n}(b) \rightarrow$ $\infty(n \rightarrow \infty)$ for every $b \in B$.

$(\mathrm{U})$ There are two $\mathcal{E}$-unicity sets $A, B$ in $\mathbb{C}^{N}$ such that $\Phi_{n}(a) \rightarrow 0$ $(n \rightarrow \infty)$ for all $a \in A$, and for each finite subset $F \subset B$ there exists an increasing sequence $\left(n_{k}\right) \subset \mathbb{N}$ with the property that for every $b \in F$ the sequence $\left(\Phi_{n_{k}}(b)\right)$ converges to a nonzero complex number.

$(\mathrm{V})$ There is an $\mathcal{E}$-unicity set $B$ in $\mathbb{C}^{N}$ such that for each finite subset $F \subset B$ there exists an increasing sequence $\left(n_{k}\right) \subset \mathbb{N}$ with the property that for every $b \in F$ the sequence $\left(\Phi_{n_{k}}(b)\right)$ converges to a nonzero complex number. In addition, $m\left(\Phi_{n}\right) \rightarrow \infty(n \rightarrow \infty)$.

(W) There are two $\mathcal{E}$-unicity sets $A, B$ in $\mathbb{C}^{N}$ such that for every $a \in A$ the sequence $\left(\Phi_{n}(a)\right)$ converges, and for every finite subset $F \subset B$ there is an increasing sequence $\left(n_{k}\right) \subset \mathbb{N}$ with $\Phi_{n_{k}}(b) \rightarrow \infty(k \rightarrow \infty)$ for all $b \in F$.

We are now ready to state our next result. In the remainder of this paper, $\Phi$ and $\Phi_{i}(i \in I:=$ an arbitrary index set) will denote entire functions of subexponential type if $G \neq C^{N}$ and of exponential type if $G=\mathbb{C}^{N}, G$ being a given domain in $\mathbb{C}^{N}$. Thus, the operators $\Phi(D), \Phi_{i}(D)(i \in I)$ are well defined on $H(G)$.

TheOREM 2.4. Suppose that $G$ is a Runge domain of $\mathbb{C}^{N}$ and that $\left(\Phi_{n}\right)$ satisfies at least one of the conditions $(\mathrm{P})-(\mathrm{W})$. Then $\mathrm{HC}\left(\left(\Phi_{n}(D)\right)\right)$ is residual in $H(G)$.

Proof. Recall that, by Lemma 2.3, the set $M(S)$ is total in $H\left(\mathbb{C}^{N}\right)$ (hence in $H(G)$, because $G$ is Runge) whenever $S$ is an $\mathcal{E}$-unicity set. Recall also that the set $\left\{z^{p}: p \in \mathbb{N}_{0}^{N}\right\}$ is total in $H(G)$, because that set spans \{polynomials\}. Take $X=H(G)$ and $T_{n}=\Phi_{n}(D)(n \in \mathbb{N})$. Apply the result mentioned just before Theorem 2.2 for $\mathcal{A}=M(A), \mathcal{B}=M(B)$ if $\left(\Phi_{n}\right)$ satisfies $(\mathrm{P})$, and for $\mathcal{A}=\left\{z^{p}: p \in \mathbb{N}_{0}^{N}\right\}, \mathcal{B}=M(B)$ if $\left(\Phi_{n}\right)$ satisfies (Q). Apply condition 
(A) of Theorem 2.2 for $\mathcal{A}=M(A), \mathcal{B}=M(B)$ if $\left(\Phi_{n}\right)$ satisfies (R), and for $\mathcal{A}=\left\{z^{p}: p \in \mathbb{N}_{0}^{N}\right\}, \mathcal{B}=M(B)$ if $\left(\Phi_{n}\right)$ satisfies (S). Apply condition (B) of Theorem 2.2 to $\mathcal{A}=M(A), \mathcal{B}=M(B)$ if $\left(\Phi_{n}\right)$ satisfies (T). Apply condition (C) of Theorem 2.2 to $\mathcal{A}=M(A), \mathcal{B}=M(B)$ if $\left(\Phi_{n}\right)$ satisfies (U), and to $\mathcal{A}=\left\{z^{p}: p \in \mathbb{N}_{0}^{N}\right\}, \mathcal{B}=M(B)$ if $\left(\Phi_{n}\right)$ satisfies (V). Finally, apply condition (D) of Theorem 2.2 to $\mathcal{A}=M(A), \mathcal{B}=M(B)$ if $\left(\Phi_{n}\right)$ satisfies (W).

Let us furnish several examples that illustrate Theorem 2.4. The reader will realize that none of these can be derived from Theorems 8, 9 of [Be3]. First, we should fix some subsets. Consider $S=\left\{n^{1 / 2}: n \in \mathbb{N}\right\}$ and let $\left(r_{j}\right)$ be any sequence of positive numbers such that the plane disks $\left\{\left|z-j^{1 / 2}\right|<r_{j}\right\} \quad(j \in \mathbb{N})$ are pairwise disjoint, for instance, $r_{j}=1 /(6 j)$. Define the compact sets $K_{n}:=\left(L_{n} \cup S\right) \cap I_{n}(n \in \mathbb{N})$, where

$$
\begin{aligned}
I_{n} & :=[-n, n] \times[-n, n], \\
L_{n} & :=\mathbb{C} \backslash\left[((0, \infty) \times(-1 / n, 0)) \cup \bigcup_{j=1}^{\infty}\left\{\left|z-j^{1 / 2}\right|<r_{j} / n\right\}\right] .
\end{aligned}
$$

It is easy to see that each $K_{n}$ has connected complement. Define the functions $f_{n}, g_{n}: K_{n} \rightarrow \mathbb{C}(n \in \mathbb{N})$ as

$$
f_{n}(z)=\left\{\begin{array}{ll}
1 & \left(z \in L_{n} \cap I_{n}\right), \\
n & \left(z \in S \cap I_{n}\right),
\end{array} \quad g_{n}(z)= \begin{cases}1 & \left(z \in L_{n} \cap I_{n}\right), \\
0 & \left(z \in S \cap I_{n}\right) .\end{cases}\right.
$$

It is clear that every $f_{n}$ and every $g_{n}$ is holomorphic on some open subset containing $K_{n}$ and depending on $n$. Then Runge's theorem guarantees the existence of polynomials $P_{n}, Q_{n}$ satisfying

$$
\left\|P_{n}-f_{n}\right\|_{K_{n}}<1 / n \text { and }\left\|Q_{n}-g_{n}\right\|_{K_{n}}<1 / n \quad(n \in \mathbb{N}) .
$$

Since $L_{n} \cap I_{n}$ and $S \cap I_{n}$ grow up to $\mathbb{C} \backslash S$ and $S$, respectively, as $n$ tends to infinity, the last two inequalities lead us to the following conclusions on point convergence: $P_{n} \rightarrow 1$ on $\mathbb{C} \backslash S, P_{n} \rightarrow \infty$ on $S, Q_{n} \rightarrow 1$ on $\mathbb{C} \backslash S$ and $Q_{n} \rightarrow 0$ on $S$ as $n \rightarrow \infty$.

EXAMPLE 1. There is a residual set of entire functions $f$ on $\mathbb{C}$ such that each entire function can be locally uniformly approximated by entire functions of the form

$$
\sum_{j=0}^{n} A_{j n} f^{(j)} \quad(n \in \mathbb{N}),
$$

where $A_{n n}=1$ and

$$
A_{j n}=(-1)^{n-j} \sum_{1 \leq i_{1}<\ldots<i_{n-j} \leq n}\left(i_{1} \ldots i_{n-j}\right)^{1 / 2} \quad(0 \leq j \leq n-1) .
$$

Indeed, it suffices to apply Theorem 2.4 with condition (P) or (T) for $A=S$, $B=\mathbb{C} \backslash S, \Phi_{n}(z)=\prod_{j=1}^{n}\left(z-j^{1 / 2}\right)(n \in \mathbb{N})$ (use Cardano-Vieta's relations). 
ExAmple 2. The set $\mathrm{HC}\left(\left(P_{n}(D)\right)\right)$ is residual in $H(\mathbb{C})$ because Theorem 2.4 can be applied with condition $(\mathrm{T})$ or $(\mathrm{W})$ for $A=\mathbb{C} \backslash S, B=S$.

ExAmple 3. The set $\mathrm{HC}\left(\left(Q_{n}(D)\right)\right)$ is residual in $H(\mathbb{C})$ because Theorem 2.4 can be applied with condition $(\mathrm{R})$ or $(\mathrm{U})$ for $A=S, B=\mathbb{C} \backslash S$.

Properties analogous to $(\mathrm{P})-(\mathrm{W})$ regarding the densely hereditary hypercyclicity of $\left(\Phi_{n}(D)\right)$ can be formulated as in [Be4, Section 3]. This would yield sufficient conditions for the existence of dense $\left(\Phi_{n}(D)\right)$-hypercyclic linear submanifolds in $H(G)$.

Birkhoff [Bir] essentially proved that given an unbounded sequence $\left(a_{n}\right) \subset \mathbb{C}$ there exists an entire function in $\mathbb{C}$ such that the set $\left\{f\left(z+a_{n}\right)\right.$ : $n \in \mathbb{N}\}$ of translates is dense in $H(\mathbb{C})$, i.e., the sequence $\left(\tau_{a_{n}}\right)$ is hypercyclic (as a matter of fact, the sequence $\left(a_{n}\right)$ depended on the particular entire function to be approximated; in [Luh] this dependence is dropped). His constructive proof can be adapted to $\mathbb{C}^{N}$ : see, for instance, [Abe] and [AbZ]; see also $[\mathrm{ArG}]$ for corresponding results for harmonic functions on $\mathbb{R}^{N}$. As a quick application of the latter theorem, we will obtain the Birkhoff theorem in several variables.

ThEOREM 2.5. Assume that $S \subset \mathbb{C}^{N}$. Then the following conditions are equivalent:

(a) $S$ is unbounded.

(b) The family $\left(\tau_{a}\right)_{a \in S}$ of operators is hypercyclic on $H\left(\mathbb{C}^{N}\right)$.

(c) $\mathrm{HC}\left(\left(\tau_{a}\right)_{a \in S}\right)$ is residual in $H\left(\mathbb{C}^{N}\right)$.

(d) $\left(\tau_{a}\right)_{a \in S}$ is not equicontinuous on $H\left(\mathbb{C}^{N}\right)$.

Proof. The implications $(\mathrm{c}) \Rightarrow(\mathrm{b}) \Rightarrow(\mathrm{d})$ are trivial. If $S$ is bounded, take $M \in(0, \infty)$ with $|a| \leq M$ for all $a \in S$. Given a basic neighbourhood $V(K, \varepsilon)$ of the origin in $H\left(\mathbb{C}^{N}\right)$, it is clear that

$$
\bigcup_{a \in S} \tau_{a}(V(L, \delta)) \subset V(K, \varepsilon),
$$

where $\delta=\varepsilon$ and $L=\{z+w: z \in K,|w| \leq M\}$. Hence $\left(\tau_{a}\right)_{a \in S}$ is equicontinuous, so (d) implies (a).

For $(\mathrm{a}) \Rightarrow(\mathrm{c})$, we will try to apply Theorem 2.4 under condition $(\mathrm{P})$ (it is also possible to use Theorem 8 of [Be3]). By hypothesis, there is a sequence $\left(a_{n}\right) \subset S$ with $a_{n} \rightarrow \infty(n \rightarrow \infty)$. Assume that $a_{n}=\left(a_{n 1}, \ldots, a_{n N}\right)$ and $b_{n j}=\operatorname{Re} a_{n j}, c_{n j}=\operatorname{Im} a_{n j}(j=1, \ldots, N ; n \in \mathbb{N})$. By taking a subsequence and possibly using a permutation of the variables $z_{1}, \ldots, z_{N}$ together with a rotation in the variable $z_{1}$ (the latter two operations generate fixed automorphisms of $H\left(\mathbb{C}^{N}\right)$ which preserve hypercyclicity), we can suppose without loss of generality that $b_{n 1} \rightarrow \infty(n \rightarrow \infty)$ and that there is a $2 N$-tuple 
$\left(\varepsilon_{1}, \delta_{1}, \ldots, \varepsilon_{N}, \delta_{N}\right) \in\{0,1\}^{2 N}$ such that the sequence $\left(a_{n}\right)$ lies in

$$
\begin{aligned}
\Pi\left(\varepsilon_{1}, \delta_{1}, \ldots, \varepsilon_{N}, \delta_{N}\right):=\left\{z=\left(b_{1}+i c_{1}, \ldots, b_{N}+i c_{N}\right) \in \mathbb{C}^{N}:\right. \\
\left.(-1)^{\varepsilon_{1}} b_{1} \geq 0,(-1)^{\delta_{1}} c_{1} \geq 0, \ldots,(-1)^{\varepsilon_{N}} b_{N} \geq 0,(-1)^{\delta_{N}} c_{N} \geq 0\right\} .
\end{aligned}
$$

Take

$$
\begin{aligned}
& A=\operatorname{int} \Pi\left(1-\varepsilon_{1}, \delta_{1}, 1-\varepsilon_{2}, \delta_{2}, \ldots, 1-\varepsilon_{N}, \delta_{N}\right), \\
& B=\operatorname{int} \Pi\left(\varepsilon_{1}, 1-\delta_{1}, \varepsilon_{2}, 1-\delta_{2}, \ldots, \varepsilon_{N}, 1-\delta_{N}\right),
\end{aligned}
$$

where "int" denotes interior. Trivially, $A$ and $B$ are nonempty open subsets of $\mathbb{C}^{N}$. Now, note that $\tau_{a_{n}}=\Phi_{n}(D)$, where $\Phi_{n}(z)=e^{a_{n} z}(n \in \mathbb{N})$. For any $z=\left(z_{1}=x_{1}+i y_{1}, \ldots, z_{N}=x_{N}+i y_{N}\right) \in \mathbb{C}^{N}$ we have

$$
\begin{aligned}
\left|\Phi_{n}(z)\right| & =\exp \left(\sum_{j=1}^{N}\left(b_{n j} x_{j}-c_{n j} y_{j}\right)\right) \\
& =\exp \left(b_{n 1} x_{1}\right) \cdot \exp \left(\sum_{j=2}^{N} b_{n j} x_{j}-\sum_{j=1}^{N} c_{n j} y_{j}\right) .
\end{aligned}
$$

Observe that $b_{n 1} x_{1} \rightarrow \infty(n \rightarrow \infty)$ and $\sum_{j=2}^{N} b_{n j} x_{j}-\sum_{j=1}^{N} c_{n j} y_{j} \geq 0$ for all $z \in B$, and $b_{n 1} x_{1} \rightarrow-\infty(n \rightarrow \infty)$ and $\sum_{j=2}^{N} b_{n j} x_{j}-\sum_{j=1}^{N} c_{n j} y_{j} \leq 0$ for all $z \in A$. Thus, $\Phi_{n} \rightarrow 0(n \rightarrow \infty)$ pointwise on $A$ and $\Phi_{n} \rightarrow \infty(n \rightarrow \infty)$ pointwise on $B$. This finishes the proof because if $\operatorname{HC}\left(\left(\tau_{a_{n}}\right)\right)$ is residual then, trivially, so is $\operatorname{HC}\left(\left(\tau_{a}\right)_{a \in S}\right)$.

Note that for $S=\{n a: n \in \mathbb{N}\}\left(a \in \mathbb{C}^{N} \backslash\{0\}\right.$ fixed), property (c) is derived from [GoS, Section 5]. This property is also obtained for an unbounded sequence $S=\left\{a_{n}: n \in \mathbb{N}\right\}$ in the case $N=1$ by a different universality proof in $[\mathrm{GeS}]$.

A slight improvement of Godefroy-Shapiro's theorem is possible as application of Theorem 2.4 by introducing a multiplicative complex sequence. Note that the condition on $\left(c_{n}\right)$ in the next result implies that $\left(\left(n !\left|c_{n}\right|\right)^{1 / n}\right)$ is unbounded (cf. Theorem 2.12), and that Godefroy-Shapiro's result is the special case $G=\mathbb{C}^{N}, c_{n} \equiv 1$ under condition (b).

Theorem 2.6. Let $\left(c_{n}\right)$ be a complex sequence. Assume that $G$ is a Runge domain in $\mathbb{C}^{N}$ and that $\Phi$ is nonconstant. Suppose that at least one of the following properties is satisfied:

(a) $\left(\left|c_{n}\right|^{1 / n}\right)$ does not converge to zero and $\Phi(0)=0$.

(b) $0<\liminf _{n \rightarrow \infty}\left|c_{n}\right|^{1 / n} \leq \limsup _{n \rightarrow \infty}\left|c_{n}\right|^{1 / n}<\infty$.

Then the set $\operatorname{HC}\left(\left(c_{n} \Phi^{n}(D)\right)\right)$ is residual in $H(G)$.

Proof. Consider the sequence of entire functions $\Phi_{n}(z)=c_{n} \Phi(z)^{n}(n \in \mathbb{N}$; $z \in \mathbb{C})$. Under the hypotheses of (a), there are $M \in(0, \infty)$ and an increasing sequence $\left(n_{k}\right)$ of positive integers with $\left|c_{n_{k}}\right| \geq M^{n_{k}}(k \in \mathbb{N})$. Since $\Phi$ is 
nonconstant, there is a nonempty open subset $B \subset \mathbb{C}^{N}$ on which $|\Phi(z)|>$ $2 / M$, hence

$$
\left|\Phi_{n_{k}}(z)\right|>2^{n_{k}} \rightarrow \infty \quad(k \rightarrow \infty)
$$

for all $z \in B$. Moreover $m\left(\Phi_{n_{k}}\right)=n_{k} \cdot m(\Phi) \rightarrow \infty(k \rightarrow \infty)$ because $m(\Phi)>0$. Consequently, Theorem 2.4 can be applied because condition (Q) is satisfied, and we are done. Under the hypothesis of (b), we have

$$
M^{n_{k}} \leq\left|c_{n_{k}}\right| \leq M_{1}^{n_{k}} \quad(k \in \mathbb{N})
$$

for some finite positive constants $M, M_{1}$ and some increasing sequence $\left(n_{k}\right) \subset \mathbb{N}$. Choose the set $B$ as in the first case. Choose also a nonempty open subset $A \subset \mathbb{C}^{N}$ on which $|\Phi(z)|<1 /\left(2 M_{1}\right)$. Then

$$
\left|\Phi_{n_{k}}(z)\right| \leq 1 / 2^{n_{k}} \rightarrow 0 \quad(k \rightarrow \infty)
$$

for all $z \in A$. Condition (P) in Theorem 2.4 is satisfied this time, and the proof is finished.

Additional sufficient conditions for hypercyclicity will be furnished later (see Theorems $2.12,2.13,2.15)$. Next, we state a necessary condition for the hypercyclicity of $\left(\Phi_{n}(D)\right)$. We need some notation first. If $a=\left(a_{1}, \ldots, a_{N}\right) \in$ $\mathbb{C}^{N}$ and $r>0$, we denote by $D(a, r)$ the closed polydisk with centre $a$ and radius $r$, i.e., $D(a, r)=\left\{z \in \mathbb{C}^{N}:\left|z_{j}-a_{j}\right| \leq r, 1 \leq j \leq N\right\}$. We consider the distance $d(z, a)=\max \left\{\left|z_{1}-a_{1}\right|, \ldots,\left|z_{N}-a_{N}\right|\right\} \quad\left(z, a \in \mathbb{C}^{N}\right)$. The inscribed radius of $G$ is $\varrho(G)=\sup _{b \in G} \inf _{a \notin G} d(a, b)=\sup \{r>0$ : there is a polydisk $D$ of radius $r$ with $D \subset G\}$. For future reference we point out here that the circumscribed radius of $G$ is defined as $R(G)=\inf _{a \in \mathbb{C}^{N}} \sup _{b \in G} d(a, b)=$ $\inf \{r>0$ : there is a polydisc $D$ of radius $r$ with $G \subset D\}$. With each sequence $\left(\Phi_{n}(z)=\sum_{|p| \geq 0} c_{p n} z^{p}\right) \subset H\left(\mathbb{C}^{N}\right)$, where each $\Phi_{n}$ is of exponential type, we associate the number in $[0, \infty]$ given by

$$
\alpha=\alpha\left(\left(\Phi_{n}\right)\right):=\limsup _{n \rightarrow \infty}\left(\sup _{|p|>0}\left(p ! \cdot\left|c_{p n}\right|\right)^{1 /|p|}\right) .
$$

Note that for each $n \in \mathbb{N}$ the number $\sup _{|p|>0}\left(p ! \cdot\left|c_{p n}\right|\right)^{1 /|p|}$ is finite.

Theorem 2.7. Suppose that $G \subset \mathbb{C}^{N}$ is a domain. Assume that $\Phi_{n}(z)=$ $\sum_{|p| \geq 0} c_{p n} z^{p}(n \in \mathbb{N})$ are entire functions such that the sequence $\left(\Phi_{n}(0)\right)$ is bounded. If the sequence $\left(\Phi_{n}(D)\right)$ is hypercyclic on $H(G)$ then $\varrho(G) \leq \alpha$.

Proof. For each $n \in \mathbb{N}$ define $K_{n}=\sup _{|p|>0}\left(p ! \cdot\left|c_{p n}\right|\right)^{1 /|p|}$, so that $\alpha=$ $\lim \sup _{n \rightarrow \infty} K_{n}$. Let $\beta \in(0, \infty)$ with $\left|c_{0 n}\right|=\left|\Phi_{n}(0)\right| \leq \beta(n \in \mathbb{N})$. Assume, by the way of contradiction, that $\lim _{\sup _{n \rightarrow \infty}} K_{n}<\varrho(G)$ and that $f \in H(G)$ is hypercyclic for $\left(\Phi_{n}(D)\right)$. Fix positive numbers $r, R$ with $\lim \sup _{n \rightarrow \infty} K_{n}<$ $r<R<\varrho(G)$. Then there exists a polydisk $D(a, R) \subset G$. By Cauchy's 
inequalities (see [Hor, Theorem 2.2.7]) we get

$$
\left|\left(D^{p} f\right)(a)\right| \leq p ! \cdot \frac{\|f\|_{D(a, R)}}{R^{|p|}} \quad(|p| \geq 0) .
$$

In addition, there is $m \in \mathbb{N}$ with $K_{n} \leq r$ for all $n \geq m$. Therefore

$$
\left|c_{p n}\right| \leq \frac{r^{|p|}}{p !} \quad(|p|>0 ; n \geq m)
$$

hence

$$
\begin{aligned}
\left|\left(\Phi_{n}(D) f\right)(a)\right|=\left|\sum_{|p| \geq 0} c_{p n} D^{p} f(a)\right| \\
\quad=\left|c_{0 n} f(a)+\sum_{|p|>0} c_{p n} D^{p} f(a)\right| \leq \beta|f(a)|+\sum_{|p|>0} \frac{r^{|p|}}{p !} \cdot p ! \cdot \frac{\|f\|_{D(a, R)}}{R^{|p|}} \\
\leq\|f\|_{D(a, R)} \cdot\left(\beta+\sum_{|p| \geq 0}(r / R)^{|p|}\right)=\|f\|_{D(a, R)} \cdot\left(\beta+\left(\frac{R}{R-r}\right)^{N}\right)
\end{aligned}
$$

for every $n \geq m$. Consequently, the sequence $\left\{\left(\Phi_{n}(D) f\right)(a): n \in \mathbb{N}\right\}$ is bounded, which is absurd. The proof is finished.

Observe that the last theorem extends Theorem 2 of [Be2], which asserted that if $G$ is a domain in $\mathbb{C}$ and $\left(c_{n} D^{n}\right)$ is hypercyclic in $H(G)$ then $\limsup _{n \rightarrow \infty}\left(n !\left|c_{n}\right|\right)^{1 / n} \geq \varrho(G)$; note that this is just the case $N=1, \Phi_{n}(z)=$ $c_{n} z^{n}$. Observe also that Theorem 2.7 implies in particular that if $\left(\Phi_{n}(D)\right)$ is hypercyclic in $H\left(\mathbb{C}^{N}\right)$ then either $\left\{\Phi_{n}(0): n \in \mathbb{N}\right\}$ or $\left\{\sup _{|p|>0}\left(p !\left|c_{p n}\right|\right)^{1 /|p|}\right.$ : $n \in \mathbb{N}\}$ is unbounded.

A corresponding sufficient condition for equicontinuity can be formulated, but the sequence $\left(\Phi_{n}(D)\right)$ may be replaced by a general family $\left\{\Phi_{i}(D)\right.$ $: i \in I\}$ of differential operators. This will be achieved in Theorem 2.9. Before this, we need a definition and an auxiliary statement. A polydomain in $\mathbb{C}^{N}$ is a product $G=G_{1} \times \ldots \times G_{N}$ of domains in $\mathbb{C}$. The following lemma is a generalization of Theorem 13.5 of [Rud]. Its proof can be made by induction and it is left to the reader.

LEMMA 2.8. If $G$ is a polydomain in $\mathbb{C}^{N}$ and $K \subset G$ is a compact subset, then there are cycles $\gamma_{1}, \ldots, \gamma_{N}$ with $\gamma_{1} \times \ldots \times \gamma_{N} \subset G \backslash K$ such that for all $f \in H(G), p=\left(p_{1}, \ldots, p_{N}\right) \in \mathbb{N}_{0}^{N}$ and $z=\left(z_{1}, \ldots, z_{N}\right) \in K$, the following Cauchy formula holds:

$$
D^{p} f(z)=\frac{p !}{(2 \pi i)^{N}} \oint_{\gamma_{1}} \ldots \oint_{\gamma_{N}} \frac{f\left(t_{1}, \ldots, t_{N}\right)}{\prod_{j=1}^{N}\left(t_{j}-z_{j}\right)^{1+p_{j}}} d t_{1} \ldots d t_{N} .
$$

Recall the following well known characterizations (see, for instance, [Boa]): an entire function $\Phi(z)=\sum_{|p| \geq 0} C_{p} z^{p}$ is of exponential type if and 
only if $\sup _{|p|>0}\left(p !\left|C_{p}\right|\right)^{1 /|p|}$ is finite, and it is of subexponential type if and only if $\lim _{|p| \rightarrow \infty}\left(p !\left|C_{p}\right|\right)^{1 /|p|}=0$.

Theorem 2.9. Suppose that $G$ is a domain in $\mathbb{C}^{N}$ and $\left\{\Phi_{i}(z)=\right.$ $\left.\sum_{|p| \geq 0} c_{p i} z^{p}: i \in I\right\}$ is a family of entire functions. Then:

(a) If $G$ is a polydomain and there is a majorant entire function $\Phi(z)=$ $\sum_{|p| \geq 0} C_{p} z^{p}$ for the family $\left(\Phi_{i}\right)$ (i.e., $C_{p} \geq 0$ and $\left|c_{p i}\right| \leq C_{p}$ for all $p \in \mathbb{N}_{0}^{N}$ and all $i \in I)$ of subexponential type then the family $\left(\Phi_{i}(D)\right)$ of operators is equicontinuous on $H(G)$.

(b) If $\left(\Phi_{i}(D)\right)$ is equicontinuous on $H(G)$ then the sequence $\left(\Phi_{i}\right)$ admits a majorant entire function of exponential type.

Proof. By the remark just above this theorem, $\left(\Phi_{i}\right)$ admits a majorant entire function of subexponential (exponential) type if and only if $\left\{c_{p i}: i\right.$ $\in I\}$ is bounded for each $p$ and $\lim _{|p| \rightarrow \infty}\left(p ! \sup _{i}\left|c_{p i}\right|\right)^{1 /|p|}=0$ (if and only if $\left\{c_{0 i}: i \in I\right\}$ is bounded and $\sup _{|p|>0, i \in I}\left(p !\left|c_{p i}\right|\right)^{1 /|p|}$ is finite, respectively). In this proof we set $T_{i}=\Phi_{i}(D)(i \in I)$.

Let us prove (a). By hypothesis, $G=G_{1} \times \ldots \times G_{N}$, where each $G_{j}$ is a domain in $\mathbb{C}$. Fix $\varepsilon>0$ and a compact subset $K \subset G$. For the corresponding basic neighbourhood $V(\varepsilon, K)$ of the origin in $H(G)$ we must find $\delta>0$ and a compact subset $L \subset G$ satisfying

$$
\bigcup_{i \in I} T_{i}(V(\delta, L)) \subset V(\varepsilon, K)
$$

Lemma 2.8 allows us to choose a "polycycle" $\gamma=\gamma_{1} \times \ldots \times \gamma_{N} \subset G \backslash K$ such that the Cauchy formula of its statement holds for $p \in \mathbb{N}_{0}^{N}, z \in K$ and $f \in H(G)$. Since $G$ is a polydomain we may suppose without loss of generality that $K=K_{1} \times \ldots \times K_{N}$, where each $K_{j}$ is a compact subset of $G_{j}$. Set

$$
\mu:=\inf \left\{\left|t_{j}-z_{j}\right|: t_{j} \in \gamma_{j} ; z_{j} \in K_{j} ; j=1, \ldots, N\right\}>0 .
$$

By hypothesis, $\lim _{|p| \rightarrow \infty}\left(p ! \sup _{i \in I}\left|c_{p i}\right|\right)^{1 /|p|}=0$, so there is $m \in \mathbb{N}$ with $\left(p !\left|c_{p i}\right|\right)^{1 /|p|} \leq \mu / 2$ for all $i \in I$ and all $p$ with $|p|>m$. But each family $\left\{c_{p i}: i \in I\right\}(|p| \leq m)$ is bounded, so there is a finite positive constant $M$ such that

$$
\frac{p !\left|c_{p i}\right| 2^{|p|}}{\mu^{|p|}} \leq M \quad(i \in I,|p| \geq 0) .
$$

Choose $L=\gamma$ and

$$
\delta=\frac{(\pi \mu)^{N} \cdot \varepsilon}{M \cdot \prod_{j=1}^{N} \operatorname{length}\left(\gamma_{j}\right)} .
$$


If $i \in I, f \in V(\delta, L)$ and $z \in K$ then

$$
\begin{aligned}
\left|\left(T_{i} f\right)(z)\right| & =\left|\sum_{|p| \geq 0} c_{p i} D^{p} f(z)\right| \\
& =\left|\sum_{|p| \geq 0} \frac{p ! c_{p i}}{(2 \pi i)^{N}} \oint_{\gamma_{1}} \ldots \oint_{\gamma_{N}} \frac{f\left(t_{1}, \ldots, t_{N}\right)}{\prod_{j=1}^{N}\left(t_{j}-z_{j}\right)^{1+p_{j}}} d t_{1} \ldots d t_{N}\right| \\
& \leq \sum_{|p| \geq 0} \frac{p !\left|c_{p i}\right|}{(2 \pi)^{N}} \cdot \frac{\delta}{\mu^{N+|p|}} \cdot \prod_{j=1}^{N} \operatorname{length}\left(\gamma_{j}\right) \\
& \leq \frac{\varepsilon}{2^{N}} \cdot \sum_{|p| \geq 0}(1 / 2)^{|p|}=\frac{\varepsilon}{2^{N}} \cdot\left(\sum_{k=0}^{\infty} 1 / 2^{k}\right)^{N}=\varepsilon .
\end{aligned}
$$

This proves (1), as required.

Now, we prove (b). Since $\left(T_{i}\right)$ is equicontinuous we can find $\delta>0$ and a compact subset $L \subset G$ such that

$$
\bigcup_{i \in I} T_{i}(V(L, \delta)) \subset V(\{a\}, 1),
$$

where $a$ is any fixed point of $G$. Consider the family of monomials $f_{p}$ $\left(p \in \mathbb{N}_{0}^{N}\right)$ given by

$$
f_{p}(z)=\delta \cdot\left(\frac{z-a}{R}\right)^{p}
$$

where $R=\sup \{|t-a|: t \in L\}$. Since $L$ can be chosen distinct from $\{a\}$, we see that $0<R<\infty$. Then $f_{p} \in V(\delta, L)$ for all $p$, whence $T_{i} f_{p} \in V(1,\{a\})$ by (2), that is,

$$
\left|\left(\Phi_{i}(D) f_{p}\right)(a)\right| \leq 1 \quad\left(i \in I, p \in \mathbb{N}_{0}^{N}\right) .
$$

$\operatorname{But}\left(\Phi_{i}(D) f_{p}\right)(a)=\sum_{|q| \geq 0} c_{q i} D^{q} f_{p}(a)=c_{p i} \delta \cdot p ! \cdot R^{-|p|}$, because $D^{q} f_{p}(z) \equiv 0$ if $|q| \geq|p|$ with $q \neq p$ and $D^{q} f_{p}(a)=0$ if $|q|<|p|$. Hence $\left|c_{p i} \delta \cdot p ! \cdot R^{-|p|}\right| \leq 1$, whence

$$
\left|c_{0 i}\right| \leq 1 / \delta \quad(i \in I)
$$

and

$$
\sup _{|p|>0, i \in I}\left(p !\left|c_{p i}\right|\right)^{1 /|p|} \leq R \cdot \sup _{|p|>0}(1 / \delta)^{1 /|p|}<\infty .
$$

The latter two inequalities show that $\left(\Phi_{i}\right)$ admits a majorant entire function of exponential type, as required. The proof is finished.

In the special case of a sequence $\left(\Phi_{n}\right)$ we get a generalization of the "if" part of [Be2, Theorem 3], because we consider the number $\alpha=\alpha\left(\left(\Phi_{n}\right)\right)$ defined before Theorem 2.7. Theorem 3 of [Be2] asserted that if $G$ is a domain in $\mathbb{C}$ and if $\limsup _{n \rightarrow \infty}\left(n !\left|c_{n}\right|\right)^{1 / n}=0$ then the family $\left(c_{n} D^{n}\right)$ is equicontinuous on $H(G)$, and the converse is true under the assumption that 
$G \neq \mathbb{C}$. Observe that its "if" part is again the particular case $\Phi_{n}(z)=c_{n} z^{n}$ of the next result.

Corollary 2.10. Assume that $G \subset \mathbb{C}^{N}$ is a polydomain, the sequence $\left(\Phi_{n}(0)\right)$ is bounded and $\alpha=0$. Then $\left(\Phi_{n}(D)\right)$ is equicontinuous on $H(G)$.

Proof. Suppose that $\Phi_{n}(z)=\sum_{|p| \geq 0} c_{p n} z^{p}(n \in \mathbb{N})$. Then $\left\{c_{0 n}: n \in \mathbb{N}\right\}$ is bounded and $\lim _{n \rightarrow \infty} \sup _{|p|>0}\left(p !\left|c_{p n}\right|\right)^{1 /|p|}=0$, from which it is easily derived that $\left\{c_{p n}: n \in \mathbb{N}\right\}$ is bounded for each multi-index $p$ and $\lim _{|p| \rightarrow \infty}\left(p ! \sup _{n}\left|c_{p n}\right|\right)^{1 /|p|}=0$ (for this, use the fact that for each fixed $n$ one has $\left(p !\left|c_{p n}\right|\right)^{1 /|p|} \rightarrow 0$ as $\left.|p| \rightarrow \infty\right)$. But this is to say that $\left(\Phi_{n}\right)$ has a majorant entire function of subexponential type, so part (a) of Theorem 2.9 yields the desired result.

For $G=\mathbb{C}^{N}$ we are able to characterize the equicontinuous families of differential operators.

THEOREM 2.11. The family $\left\{\Phi_{i}(D): i \in I\right\}$ of operators is equicontinuous on $H\left(\mathbb{C}^{N}\right)$ if and only if $\left(\Phi_{i}\right)$ admits a majorant entire function of exponential type.

Proof. The "only if" part is due to Theorem 2.9(b). For the converse, we can follow step by step the proof of part (a) of Theorem 2.9 with the sole exception that we may choose the polycycle $\gamma$ far enough from the compact set $K$ (so $\mu$ can be chosen as large as desired) in such a way that

$$
\sup _{|p|>0, i \in I}\left(p !\left|c_{p i}\right|\right)^{1 /|p|} \leq \mu / 2 .
$$

The constant $M$ may be chosen as $M=\max \left\{1, \sup _{i \in I}\left|c_{0 i}\right|\right\}$. The proof is finished.

In $[$ Be2, Theorem 1] it has been established that if $G \subset \mathbb{C}$ is a simply connected domain and $\left(c_{n}\right)$ is a complex sequence with

$$
R(G) \leq \limsup _{n \rightarrow \infty}\left(n !\left|c_{n}\right|\right)^{1 / n}
$$

then $\mathrm{HC}\left(\left(c_{n} D^{n}\right)\right)$ is residual in $H(G)$. A slight generalization can be obtained in the $N$-dimensional case. The proof is very similar to the 1 -dimensional case, so we omit it.

Theorem 2.12. Assume that $G \subset \mathbb{C}^{N}$ is a Runge domain and that $(p(n))$ is a sequence of multi-indices with $|p(n)| \rightarrow \infty(n \rightarrow \infty)$. If $\left(c_{n}\right)$ is a complex sequence with

$$
R(G) \leq \limsup _{n \rightarrow \infty}\left(p(n) !\left|c_{n}\right|\right)^{1 /|p(n)|}
$$

then the set $\mathrm{HC}\left(\left(c_{n} D^{p(n)}\right)\right)$ is residual in $H(G)$. 
As a consequence of Theorems 2.11, 2.12 we can get a characterization of equicontinuity and hypercyclicity of the same sequence in $H\left(\mathbb{C}^{N}\right)$. This is achieved in the next result, which in turn is an $N$-dimensional extension of [Be2, Theorem 4] (see also [Be1]).

Theorem 2.13. Assume that $\left(c_{n}\right)$ is a complex sequence and $(p(n))$ is a sequence of nonzero multi-indices such that $|p(n)| \rightarrow \infty(n \rightarrow \infty)$. Then the following properties are equivalent:

(a) The sequence $\left(\left(p(n) !\left|c_{n}\right|\right)^{1 /|p(n)|}\right)$ is bounded.

(b) There is no hypercyclic entire function for $\left(c_{n} D^{p(n)}\right)$.

(c) The set $\mathrm{HC}\left(\left(c_{n} D^{p(n)}\right)\right)$ is not residual in $H\left(\mathbb{C}^{N}\right)$.

(d) The sequence $\left(c_{n} D^{p(n)}\right)$ is equicontinuous on $H\left(\mathbb{C}^{N}\right)$.

Proof. It is evident that (b) implies (c) and that (d) implies (b). Since $R\left(\mathbb{C}^{N}\right)=\infty$, we see from Theorem 2.12 that (c) implies (a). Assume that (a) holds. Then we can apply Theorem 2.11 with $I=\mathbb{N}$ and $\Phi_{n}(z)=c_{n} z^{p(n)}$. Indeed, there is a constant $M$ with $p(n) !\left|c_{n}\right| \leq M^{|p(n)|}$ for all $n \in \mathbb{N}$, hence the function

$$
\Phi(z)=\sum_{n=1}^{\infty} \frac{M^{|p(n)|}}{p(n) !} z^{p(n)} \quad\left(z \in \mathbb{C}^{N}\right)
$$

is a majorant entire function for $\left(\Phi_{n}\right)$ of exponential type. Then $(\mathrm{d})$ is true and the proof is finished.

We point out that in [Gr2, Corollary to Theorem 4] the hypercyclicity part of $[\mathrm{Be} 2$, Theorem 4] is extended for $N=1$ to sequences of weighted pseudo-shifts in the space $H(\mathbb{C})$.

The "only if" part of [Be2, Theorem 3] can be extended in the same way to the $N$-dimensional case, as the following theorem shows.

TheOREM 2.14. Let $G=G_{1} \times \ldots \times G_{N} \subset \mathbb{C}^{N}$ be a polydomain with $G_{j}$ $\neq \mathbb{C}(j=1, \ldots, N)$. Assume that $\left(c_{n}\right)$ is a complex sequence and that $(p(n))$ is a sequence of nonzero multi-indices such that the sequence $\left(c_{n} D^{p(n)}\right)$ of operators is equicontinuous on $H(G)$. Then

$$
\lim _{n \rightarrow \infty}\left(p(n) !\left|c_{n}\right|\right)^{1 /|p(n)|}=0 .
$$

Proof. Set $\alpha:=\lim \sup _{n \rightarrow \infty}\left(p(n) !\left|c_{n}\right|\right)^{1 /|p(n)|}$. By the way of contradiction, assume that $\alpha>0$. Fix $a=\left(a_{1}, \ldots, a_{N}\right) \in G$. Then $a_{j} \in G_{j}$ and there exist $b_{j} \in \mathbb{C} \backslash G_{j}(j=1, \ldots, N)$ such that $\left|a_{j}-b_{j}\right|=\inf \left\{\left|a_{j}-t\right|: t \in \mathbb{C} \backslash G_{j}\right\}$. Define $R=\min \left\{\left|a_{j}-b_{j}\right|: j=1, \ldots, n\right\}>0$. Fix $r \in(0, R)$ with $R-r<\alpha$. Put $K=D(a, r)$. Then $K$ is a compact subset of $G$. Let $L$ be any compact subset of $G$ and $\delta$ a positive number. Let $m>0$ be so small that

$$
\frac{m}{\left(\inf \left\{\left|z_{j}-b_{j}\right|: j \in\{1, \ldots, N\}, z=\left(z_{1}, \ldots, z_{n}\right) \in L \cup K\right\}\right)^{N}}<\delta .
$$


Consider the function

$$
f(z)=\frac{m}{\prod_{j=1}^{N}\left(z_{j}-b_{j}\right)} .
$$

Then $f \in H(G)$ and, in addition, $f$ belongs to $V(\delta, L)$. Furthermore, for $z=\left(z_{1}, \ldots, z_{N}\right) \in G$,

$$
\left|\left(T_{n} f\right)(z)\right|=\frac{p(n) ! m\left|c_{n}\right|}{\prod_{j=1}^{N}\left|z_{j}-b_{j}\right|^{1+p_{j}(n)}},
$$

where $p(n)=\left(p_{1}(n), \ldots, p_{N}(n)\right)$ and $T_{n}=c_{n} D^{p(n)}$. Since $\inf \left\{\left|t-b_{j}\right|\right.$ : $\left.\left|t-a_{j}\right|<r\right\} \geq R-r$ for every $j \in\{1, \ldots, N\}$, we get

$$
\sup \left\{\left|\left(T_{n} f\right)(z)\right|: z \in K\right\} \leq \frac{p(n) !\left|c_{n}\right| m}{(R-r)^{N+|p(n)|}}=\frac{m}{(R-r)^{N}} \cdot \frac{p(n) !\left|c_{n}\right|}{(R-r)^{|p(n)|}} .
$$

But $p\left(n_{k}\right) !\left|c_{n_{k}}\right| /(R-r)^{\left|p\left(n_{k}\right)\right|} \rightarrow \infty(k \rightarrow \infty)$ for some increasing sequence $\left(n_{k}\right) \subset \mathbb{N}$, because $\alpha>R-r$. Hence $\sup \left\{\left|T_{n} f(z)\right|: z \in K\right\}=\infty$. Therefore

$$
\bigcup_{n \in \mathbb{N}} T_{n}(V(\delta, L)) \not \subset V(1, K),
$$

which implies that $\left(T_{n}\right)$ is not equicontinuous. The proof is finished.

Our final result comes back to hypercyclicity and looks slightly different from the others. It puts the emphasis on the first nonzero Taylor coefficient of each $\Phi_{n}$. This time the setting is the complex plane $\mathbb{C}$. Observe that MacLane's theorem is again recovered if we choose $\Phi_{n}(z)=z^{n}$ for each $n \in \mathbb{N}$.

THEOREM 2.15. Assume that $\left(\Phi_{n}(z)=\sum_{j=0}^{\infty} c_{j n} z^{j}\right)$ is a sequence of nonzero entire functions and set $p(n):=m\left(\Phi_{n}\right)(n \in \mathbb{N})$. Assume that the following three conditions are fulfilled:

(a) $p(n) \rightarrow \infty$ as $n \rightarrow \infty$.

(b) $p(n)\left|c_{p(n), n}\right|^{k / p(n)} \rightarrow \infty$ as $n \rightarrow \infty$ for every $k \in \mathbb{N}$.

(c) Each sequence $\left\{c_{j+p(n), n}: n \in \mathbb{N}\right\}(j \in \mathbb{N})$ is bounded.

Then the set $\operatorname{HC}\left(\left(\Phi_{n}(D)\right)\right)$ is residual in $H(G)$ for any simply connected domain $G \subset \mathbb{C}$.

Proof. We wish to apply Lemma 2.1 with $X=H(G)=Y, X_{0}=$ \{polynomials $\}=Y_{0}$ and $T_{n}=\Phi_{n}(D)(n \in \mathbb{N})$. If $P$ is a polynomial then by (a) there exists $n_{0} \in \mathbb{N}$ with $p(n)>\operatorname{deg}(P)$ for all $n \geq n_{0}$, hence $D^{j} P=0$ for all $j \geq p(n)\left(n \geq n_{0}\right)$. Therefore $T_{n} P=0$ eventually and condition (a) of Lemma 2.1 is satisfied. Now fix $m$ and $n$ in $\mathbb{N}$ and try to solve the equation $T_{n} f=z^{m}$. Observe that $T_{n}=\Psi_{n}(D) \circ D^{p(n)}$, where $\Psi_{n}(z)=\sum_{j=0}^{\infty} a_{j n} z^{j}$ and $a_{j n}=c_{j+p(n), n}$, so $a_{0 n} \neq 0$ for all $n \in \mathbb{N}$. Consider the equation

$$
\Psi_{n}(D) g=z^{m},
$$


where $g$ is a polynomial of degree not greater than $m$, say, $g(z)=\sum_{k=0}^{m} b_{k n} z^{k}$. It is easy to see that such a polynomial solution exists. Indeed, (1) is equivalent to

$$
\sum_{j=0}^{m} a_{j n}\left(\sum_{k=0}^{m} b_{k n} z^{k}\right)^{(j)}=z^{m}
$$

which in turn is the same as the system

$$
\left\{\begin{array}{l}
\sum_{j=k}^{m} a_{j-k, n} b_{j n} \cdot \frac{j !}{k !}=0 \quad(k=0,1, \ldots, m-1), \\
a_{0 n} b_{m n}=1 .
\end{array}\right.
$$

This is a recurrent square system with determinant $a_{0 n}^{m+1} \neq 0$, so it has a unique solution $\left(b_{0 n}, \ldots, b_{m n}\right)$ and Cramer's rule yields

$$
b_{k n}=\frac{1}{a_{0 n}^{m+1}} \cdot \sum_{j=1}^{m} P_{j k m}\left(a_{1 n}, \ldots, a_{m n}\right) a_{0 n}^{j}
$$

for $k \in\{0,1, \ldots, m\}$, where $P_{j k m}(j=1, \ldots, m)$ are polynomials of $m$ complex variables not depending on $n$. From (c), there is a finite positive constant $M$, which does not depend on $n$, such that

$$
\left|P_{j k m}\left(a_{1 n}, \ldots, a_{m n}\right)\right| \leq M
$$

for all $k \in\{0,1, \ldots, m\}$ and all $j \in\{1, \ldots, m\}$. Hence a solution of $T_{n} f=z^{m}$ is

$$
f(z)=f_{n}(z)=\sum_{k=0}^{m} b_{k n} \frac{z^{k+p(n)}}{(k+p(n)) !} \quad(n \in \mathbb{N}),
$$

where $b_{k n}$ is given by (2). Fix $R>1$. Then from (3), for $|z| \leq R$ we deduce that

$$
\left|f_{n}(z)\right| \leq(m+1) \sum_{j=1}^{m} \frac{M R^{m}}{\left|a_{0 n}\right|^{m+1-j}} \cdot \frac{R^{p(n)}}{p(n) !} \rightarrow 0 \quad(n \rightarrow \infty)
$$

since (b) and Stirling's formula lead to

$$
\left(p(n) !\left|a_{0 n}\right|^{m+1-j}\right)^{1 / p(n)} \rightarrow \infty \quad(n \rightarrow \infty),
$$

so the terms of the latter sequence are eventually greater than, for instance, $1 /(2 R)$. Therefore $\left(f_{n}\right)$ tends to zero in $H(G)$. The proof for the case $m=0$ is easier and left to the reader. Define

$$
S_{n}\left(z^{m}\right):=f_{n}(z) \quad\left(m \in \mathbb{N}_{0} ; n \in \mathbb{N}\right)
$$

and extend $S_{n}$ to $Y_{0}$ by linearity. Then it is clear that $S_{n} P \rightarrow 0(n \rightarrow \infty)$ and $T_{n}\left(S_{n} P\right)=P \rightarrow P$ as $n \rightarrow \infty$. Consequently, conditions (b) and (c) in Lemma 2.1 are also fulfilled, as required. 
For instance, there is an entire function $f$ in $\mathbb{C}$ with the property that any entire function can be locally uniformly approximated by functions of the form

$$
c_{n}\left(f^{(n)}+f^{(n+1)}\right) \quad(n \in \mathbb{N}),
$$

where $c_{n}=n^{-n /(\log n)^{1 / 2}}$. Indeed, the sequence $\left\{\Phi_{n}(z)=c_{n} z^{n}(1+z)\right\}$ satisfies all hypotheses of the latter theorem, because $\left(c_{n}\right)$ is bounded, $p(n) \rightarrow \infty$ and $p(n) \cdot n^{-k n /\left(p(n)(\log n)^{1 / 2}\right)} \rightarrow \infty(n \rightarrow \infty)$ for all $k \in \mathbb{N}$, where $p(n) \equiv n$ here. Note that this example shows that Theorem 2.15 is not included in Theorem 2.4: in fact, $\Phi_{n}(z) \rightarrow 0$ as $n \rightarrow \infty$ for all $z \in \mathbb{C}$, hence the $\mathcal{E}$-unicity set $B$ is not available in order to apply Theorem 2.4 .

\section{References}

[Abe] Y. Abe, Universal holomorphic functions in several variables, Analysis 17 (1997), $71-77$.

[AbZ] Y. Abe and P. Zappa, Universal functions in complex general groups, J. Approx. Theory 100 (1999), 221-232.

[ArG] D. H. Armitage and P. M. Gauthier, Recent developments in harmonic approximation, with applications, Results Math. 29 (1996), 1-15.

[Ans] S. I. Ansari, Existence of hypercyclic operators on topological vector spaces, J. Funct. Anal. 148 (1997), 384-390.

[Be1] L. Bernal-González, Una nota sobre sucesiones complejas y equicontinuidad de operadores, Rev. Roumaine Math. Pures Appl. 34 (1989), 643-645.

[Be2] - Derivative and antiderivative operators and the size of complex domains, Ann. Polon. Math. 59 (1994), 267-274.

[Be3] - Hypercyclic sequences of differential and antidifferential operators, J. Approx. Theory 96 (1999), 323-337.

[Be4] - Densely hereditarily hypercyclic sequences and large hypercyclic manifolds, Proc. Amer. Math. Soc. 127 (1999), 3279-3285.

[Bes] J. P. Bès, Invariant manifolds of hypercyclic vectors for the real scalar case, ibid. 127 (1999), 1801-1804.

[Bir] G. D. Birkhoff, Démonstration d'un théorème élémentaire sur les fonctions entières, C. R. Acad. Sci. Paris 189 (1929), 473-475.

[Boa] R. P. Boas, Entire Functions, Academic Press, New York, 1954.

[Bou] P. Bourdon, Invariant manifolds of hypercyclic operators, Proc. Amer. Math. Soc. 118 (1993), 845-847.

[Cal] M. C. Calderón-Moreno, Universality of derivative and antiderivative operators with holomorphic coefficients, Ann. Polon. Math., to appear.

[Dic] D. G. Dickson, Expansions in series of solutions of linear difference-differential and infinite order differential equations with constant coefficients, Mem. Amer. Math. Soc. 23 (1957).

[GeS] G. Gethner and J. H. Shapiro, Universal vectors for operators on spaces of holomorphic functions, Proc. Amer. Math. Soc. 100 (1987), 281-288.

[GoS] G. Godefroy and J. H. Shapiro, Operators with dense, invariant, cyclic vector manifolds, J. Funct. Anal. 98 (1991), 229-269. 
[Gr1] K. G. Grosse-Erdmann, Universal families and hypercyclic operators, Bull. Amer. Math. Soc. 36 (1999), 345-381.

[Gr2] - Hypercyclic and chaotic weighted shifts, Studia Math. 139 (2000), 47-68.

[Her] D. Herrero, Limits of hypercyclic and supercyclic operators, J. Funct. Anal. 99 (1991), 179-190.

[Hor] L. Hörmander, An Introduction to Complex Analysis in Several Variables, NorthHolland, Amsterdam, 1973.

[Kra] S. G. Krantz, Function Theory of Several Complex Variables, Wiley, New York, 1982.

[Luh] W. Luh, On universal functions, in: Colloq. Math. Soc. János Bolyai 19, NorthHolland, 1976, 503-511.

[Mac] G. R. MacLane, Sequences of derivatives and normal families, J. Anal. Math. 2 (1952), 72-87.

[Rud] W. Rudin, Real and Complex Analysis, 2nd. ed., Tata McGraw-Hill, Faridabad, 1974.

[Val] G. Valiron, Sur les solutions des équations différentielles linéaires d'ordre infini et à coefficients constants, Ann. École Norm. Sup. (3) 46 (1929), 25-53.

Departamento de Análisis Matemático

Facultad de Matemáticas, Apdo. 1160

Avenida Reina Mercedes

41080 Sevilla, Spain

E-mail: lbernal@cica.es

tendero@cica.es 